\title{
Elección de la Época de Plantación de la Papa en el Estado Mérida, Venezuela, en Función de la Simulación del Balance Hídrico del Cultivo
}

\author{
Gladys I. Ramos Carranza ${ }^{1}$
}

\begin{abstract}
Resumen
Se seleccionaron seis localidades productoras de papa en el Estado Mérida, Venezuela, con el propósito de determinar a través de un modelo de simulación, cuál es la época del año que reúne las mejores condiciones climáticas para iniciar la siembra. El modelo utilizado fue el de Sellers, el cual requiere información de clima (precipitación y evaporación), de suelos (textura, humedad a CC y PMP y el valor del coeficiente hidrodinámico), y del cultivo (cobertura, altura, profundidad y densidad del sistema radical).

El modelo simula el índice de productividad mensual, durante todo el año, permitiendo al usuario, seleccionar el momento de plantación seguido de un período de 90 a 120 días, en el cual el suplemento hídrico es adecuado para el crecimiento y desarrollo de todo el ciclo de la variedad elegida.

El método genera el valor (ET/ETX), que oscila entre 0 y 1 . El valor máximo uno, expresa la posibilidad de que la planta pueda evapotranspirar a la tasa máxima lo que conlleva a un crecimiento vegetativo óptimo y una mayor productividad.

En las localidades analizadas, se encontró que los meses con valores de ET/ETX apropiados para la siembra de papa son: en Mucuchíes, (abril a agosto), Timotes (octubre), Pueblo Llano (abril a julio), Bailadores (octubre), Mucurubá (abril a julio) y en Canaguá (abril a septiembre). Los meses que conforman estos períodos, están precedidos por épocas con una precipitación suficiente para mantener un contenido de humedad adecuado en la zona radicular típica para el cultivo de la papa.
\end{abstract}

Aceptado para publicación: abril 1999.

1 FONAIAP. Apartado 425, Mérida, Venezuela. 
Palabras claves adicionales: Época de plantación, índice de productividad, balance hídrico, tasa de transpiración.

\title{
Determining Planting Date in the State of Mérida, Venezuela, Based on Crop Water Balance
}

\begin{abstract}
Summary
Six potato production areas were chosen in the State of Mérida with the purpose of determining, by a simulation model, which season of the year has the best climatic conditions for crop growth. The Sellers model was used. The model requires information about weather (rainfall and evaporation), soil (texture and humidity in CC and PMP and the coefficient hydrodynamic value) crop characteristics (area covered, height, depth and the density of the root system).

The model simulated a monthly productivity index, for the whole year, allowing the user to select planting time followed by a period of 90 to 120 days, with an adequate water supply for crop growth and development.

The model generates the ET/ETX índex, ranging between 0 and 1 . The maximum value of 1 showed crop possibilities to evapotranspire at a maximum level achieving an optimum vegetative growth and greater productivity.

In the areas analized, it was found that the months with the best ET/ETX value for crop growth are: in Mucuchies (April to August); Timotes (October); Pueblo Llano (April to July); Bailadores (October); Mucuruba (April to July); and in Canagua (April to September). The months comprising those periods were preceeded by seasons with enough rainfall to maintain adequate amounts of soil water at the root zone.
\end{abstract}

Additional index words: Potato planting, productivity index, hydric balance, transpiration rate. 


\section{Introducción}

Una forma de minimizar los riesgos en la producción de cultivos, es la selección de una época de plantación favorable que permita adecuar sus requerimientos y sincronizar sus fases fenológicas con la integración de las modalidades diarias y anuales del clima, frecuentes dentro de una región dada. La época de plantación para el cultivo de la papa, depende de los factores o elementos climáticos de cada sitio, por lo tanto se justifica recabar información de cada localidad para definir períodos de inicio del ciclo del cultivo y la aplicación apropiada de labores agronómicas, garantizando la máxima suplementación hídrica a fin de satisfacer la tasa máxima de transpiración $(5,6,8,9)$.

Reviste particular interés la determinación de una apropiada época de plantación para la papa en el estado Marida, debido a las irregularidades topográficas que caracterizan esta entidad, por ser limitada la superficie apta para este cultivo en Venezuela, por la incidencia de fenómenos naturales como las heladas que caracterizan en forma determinante el microclima de algunas áreas de siembra y por constituir este rubro el de mayor aporte al valor de la producción agrícola en el estado con un $24,66 \%$ (12).

Es necesario considerar como antecedente para la selección del período de siembra, que durante los meses de abundantes precipitaciones, los riesgos de escorrentía superficial y deslizamiento en las áreas sembradas, causan desastres naturales de gran magnitud. Así mismo las áreas restringidas por legislación de parques nacionales, conllevan a un gran número de pequeños productores a establecer el cultivo en zonas de alto riesgo, incrementando las pérdidas. También juega un papel importante la variación de precios del producto y el tipo de consumo del mismo, lo que hace que estas fluctuaciones sean leves o muy notorias.

El objetivo de este estudio, consistió en determinar épocas de plantación adecuadas para el cultivo de la papa en varias localidades del estado Mérida, Venezuela, mediante el uso del índice de productividad mensual ET/ETX, a través del modelo de Balance Hídrico de Sellers el cual involucra la relación agua - suelo - planta -atmósfera, es de fácil aplicación y la información que requiere para su ejecución es de fácil acceso. 


\section{Materiales y Métodos}

El presente estudio se desarrolló para las localidades de Mucuchíes, Mucurubá, Timotes, Pueblo Llano, Bailadores y Canaguá en el estado Mérida, Venezuela (Tabla 1).

Tabla 1. Ubicación geográfica y altitudinal de las localidades analizadas. *

\begin{tabular}{|c|c|c|c|c|c|}
\hline $\begin{array}{l}\text { P. anual } \\
(\mathrm{mm})\end{array}$ & Localidad & $\begin{array}{c}\text { Altitud } \\
\text { (msnm) }\end{array}$ & $\begin{array}{l}\text { Latitud } \\
\mathrm{N}\end{array}$ & $\begin{array}{c}\text { Longitud } \\
\text { W }\end{array}$ & $\begin{array}{l}\text { Período de } \\
\text { registro (años) }\end{array}$ \\
\hline 594.2 & Mucuchíes & 3100 & 089 45'04" & 70a 55'03" & $51-91$ \\
\hline 820.7 & Mucurubá & 2320 & 08S 42' 22" & 7QS 59' 33" & $63-83$ \\
\hline 1153.2 & Pueblo Llano & 2230 & 08S 55' 25" & 70Q 39' 30" & $70-83$ \\
\hline 735.1 & Timotes & 2089 & 08959' 14" & $70 Q$ 44' $20 "$ & $53-73$ \\
\hline 685.5 & Bailadores & 1736 & 08e15' 10" & $71^{\circ}-49^{\prime} 37^{\prime \prime}$ & $68-86$ \\
\hline 1356.9 & Canaguá & 1560 & $08=08^{\prime} 08^{\prime \prime}$ & 71 s 26' 25" & $68-86$ \\
\hline
\end{tabular}

* MARNR - Zona 12. Mérida. Venezuela.

La época de plantación para cada localidad se determinó utilizando el modelo de Sellers, el cual se basa en un balance hídrico que es la formulación matemática de la Ley de conservación de la materia, aplicada al agua en un sistema determinado, que puede ser el volumen de suelo ocupado por una planta, un lote de terreno o una cuenca hidrológica (6).

El balance hídrico especifica que el total de agua que penetra en un sistema dado debe ser igual al agua que sale de él, en este caso por evapotranspiración y escurrimiento, más la diferencia entre los contenidos final e inicial (5), quedando expresado en la siguiente ecuación:

$P=E T+S+(L F-L I)$

Donde,

$\mathrm{P}=$ precipitación.

$\mathrm{ET}=$ evapotranspiración. 


$$
\begin{aligned}
& \mathrm{S}=\text { escurrimiento de agua (superficial y subterráneo). } \\
& \mathrm{LF}=\text { lámina remanente al final del período. } \\
& \mathrm{LI}=\text { lámina de agua al iniciarse al período. }
\end{aligned}
$$

Para alimentar el modelo se utilizó información básica del clima, considerándose el valor promedio mensual de la precipitación $(\mathrm{cm})$, correspondiente a un período mayor de 10 años y de la evaporación mensual de recipientes tipo A (13). Asimismo, la información básica del suelo como la textura predominante por localidad, se obtuvo de los resúmenes de los análisis de suelos del Laboratorio de Servicio del FONAIAP - Mérida (86 - 91), promedió de 6 años. Se puso énfasis en los resultados de las muestras y su caracterización en cuanto a texturas gruesas $(a, a f, f a)$, medias $(f, f a, F L, F A, A a)$ y finas $(A, A L$, $\mathrm{FAL})(11)$

El almacenamiento de agua a Capacidad de Campo (CC) y el Punto de Marchitez Permanente (PMP) en función de las clases texturales fue obtenido de la Tabla 2, donde se resumen las propiedades físicas del suelo y se efectuaron interpolaciones de los valores expresados según el predominio de cada clase textural. Estas características permiten determinar la fracción de agua aprovechable por la planta que puede ser almacenada según la textura del suelo.

Tabla 2. Resumen de las propiedades físicas del suelo.

\begin{tabular}{|l|c|c|c|c|c|}
\hline $\begin{array}{c}\text { Textura del } \\
\text { suelo }\end{array}$ & $\begin{array}{c}\text { Densidad } \\
\text { aparente } \\
\text { g/cm }\end{array}$ & $\%$ & $\begin{array}{c}\text { Cap. de } \\
\text { campo Cc } \\
\text { cm/cm }\end{array}$ & $\%$ & $\begin{array}{c}\text { Punto de marchitez } \\
\text { permanente PMP } \\
\text { cm/cm }\end{array}$ \\
\hline Arenoso & $(1.55-1.80)$ & 9 & 0.1485 & 4 & 0.066 \\
F. arenoso & $(1.40-1.60)$ & 14 & 0.2100 & 6 & 0.090 \\
Franco & $(1.35-1.50)$ & 22 & 0.3880 & 10 & 0.140 \\
F. arcilloso & $(1.30-1.40)$ & 27 & 0.3640 & 13 & 0.175 \\
Arenoso & $(1.25-1.35)$ & 31 & 0.4030 & 15 & 0.195 \\
Arcilloso & $(1.20-1.30)$ & 35 & 0.4370 & 17 & 0.213 \\
\hline
\end{tabular}

Norero, A.. CIDIAT, Mérida, Venezuela. (5).

El coeficiente hidrodinámico del suelo "s", el cual relaciona la clase textural con la restricción de humedad disponible, varia entre 0.2 y 0.6 . Este valor "s" aumenta cuando el contenido de humedad disponible, disminuye $(4,5,6,7)$. 
En cuanto a las características del cultivo se consideró el follaje, área foliar, tipo de cobertura y sistema radicular, aspectos que determinan la efectividad en el uso del agua y su flujo a través del vegetal. $(5,6)$. Se recopiló información sobre los siguientes datos: coeficiente de densidad de enraizamíento "r", que es una característica fitométrica del cultivo y oscila entre 0.2 para cultivo de raíces poco densas y 1.0 para los de raíces muy profusas. En el caso del cultivo de papa se seleccionó $r=0.8$.

La profundidad radicular típica "Rx", expresa la profundidad del área de exploración de las raíces y está en función de la textura del suelo $(4,5,7$, 8). El coeficiente de frondosidad "f", está relacionado con la altura del cultivo y su área foliar efectiva $(1,2,5)$.

En cuanto al área cultivada, se utilizaron los registros de superficie sembrada promedio mensual con el cultivo de papa en cada localidad evaluada, correspondiendo estos registros al período 1987 - 1991, con base en las estadísticas del sector agrícola del estado a excepción de Mucurubá y Canaguá por no estar disponibles. Lo anterior se realizó con el propósito de conocer la época del año en la cual se establece la mayor superficie con papa en cada localidad.

\section{Procedimiento}

Con la información anterior se alimentó el modelo de Balance hídrico de Sellers $(9,10)$ con el fin de determinar los siguientes parámetros:

- Evapotranspiración máxima (ETX):

$$
E T X=E v^{*} f
$$

Donde,

$\mathrm{Ev}=$ evaporación de recipientes tipo $\mathrm{A}$.

$f=$ coeficiente de frondosidad del cultivo.

* Coeficiente de marchitamiento incipiente $(U)$ :

$$
U=\sqrt[3]{s . r .(1-E T X \quad / N)}
$$

Donde, $\mathrm{s}=$ coeficiente hidrodinámico del suelo.

$r=$ coeficiente de densidad de enraizamiento. 
$\mathrm{N}$ = número de días del mes.

* Lámina a la cual se produce el marchitamiento incipiente (Lx):

$$
L x=L e-(U \text { * LA) }
$$

Donde,

Le = lámina de agua a capacidad de campo para iniciar el balance. LA = lámina almacenable y disponible entre el contenido a capacidad de campo y punto de marchitez.

* Coeficiente de escurrimiento neto de agua (e):

$$
\text { e }-(0.4 \text { * P2) / (ETX + P) * LC }
$$

Donde,

$\mathrm{P}$ - precipitación media mensual.

* Lámina almacenada al final del mes (LFx):

$$
\mathrm{OLFX}=(P-E T X+(1-e) \operatorname{LI}) /(1+e)
$$

Donde,

LI = Lámina presente en el suelo al iniciarse el balance.

* Coeficiente de aprovechamiento de agua (A):

$$
A=E T X /[2 \operatorname{LA}(1-U)]
$$

* Humedad final del mes bajo uso restringido de agua (LF):

$$
L F-\{P+[(1-e) L I]+[A(2 \text { LM - LI) }]\} /(1+e+A)
$$

Donde,

LM = contenido de humedad del suelo a PMP.

* Escurrimiento neto de agua (SS):

$$
\mathrm{SS}=\mathrm{e}(\mathrm{LI}+\mathrm{LF})
$$

* Evapotranspiración mensual (ET): 


$$
\mathrm{ET}=[(\mathrm{LP}-\mathrm{LM}) /(\mathrm{LX}-\mathrm{LM})] \mathrm{ETX}
$$

Donde,

LP = lámina que representa el 50\% del agua útil.

* índice de productividad mensual (ET/ETX)

\section{Resultados y Discusión}

El índice ET /ETX fue calculado para cada mes y su valor osciló entre cero y uno, siendo uno el valor indicativo de que el cultivo está evapotranspirando sin restricciones y es posible obtener el máximo rendimiento si están controlados los aspectos nutricionales y fitosanitarios y se han tomado previsiones sobre los fenómenos naturales comunes en la zona de páramo como la ocurrencia de heladas $(1,3,8,9)$.

Se selecciona como épocas de plantación para cada localidad, aquellas donde el valor del índice de productividad estuviese próximo o igual a (1) y que los tres o cuatro meses subsiguientes, también presenta valores en este mismo orden. De esta forma, se garantiza un adecuado suministro de humedad durante todo el ciclo del cultivo.

Asimismo, la salida del modelo presenta la superposición de un gráfico de los valores mensuales de ET/ETX con el de la superficie promedio sembrada por mes en la localidad analizada. Lo anterior permite comparar cuál es la época en la que el productor acostumbra a sembrar la semilla de papa y si estas fechas coinciden con las épocas en las cuales los índices ET/ETX presentan su máximo valor.

En la localidad de Mucuchíes (Figura 1), los valores ET/ETX, comienzan a incrementarse a partir del mes de abril y esta tendencia prevalece hasta finales de noviembre, cuando se inicia la estación invernal seca y la precipitación es menor que la evaporación. Tradicionalmente la mayoría de los productores de papa de esta localidad, inician labores de establecimiento del cultivo desde el mes de marzo, a partir del cual disminuyen las nuevas áreas incorporadas a la siembra hasta julio y se hace insignificante desde agosto hasta febrero del año siguiente. El establecimiento del cultivo a partir de marzo, garantiza un buen abastecimiento de agua durante todo el ciclo (3 a 4 meses), condición que beneficiaría a todas las plantaciones que se inicien, inclusive hasta el mes de junio. A partir del mes de julio, existirá buen suplemento hídrico pero se inician los riesgos de heladas que podrían afectar al cultivo en su última fase fonológica. Esta condición de temperatura que caracteriza el microclima local, es un fenómeno natural con un 100\% de probabilidad de ocurrencia el cual no puede ser ignorado al 
seleccionar la época de plantación en papa. (14) En consecuencia, es recomendable considerar la variedad de papa que se va a establecer y definir el final de su ciclo para prevenir quemado prematuro del follaje por efecto de las bajas temperaturas.

En la localidad de Pueblo Llano (Figura 2), tradicionalmente se presentan dos temporadas altas de siembra como son los meses de febrero-marzo y agosto. Estas temporadas, y los tres meses subsiguientes a cada una, necesarios para completar el ciclo de variedades de papa entre 90 y 120 días, garantizan valores adecuados del índice ET /ETX para satisfacer las exigencias del cultivo.

En la Figura 3, se presentan las épocas tradicionales de siembra en Bailadores, donde se observa la tendencia a sembrar la mayor superficie durante los meses de enero y febrero, a partir de los cuales se incrementa el índice ET/ETX y se presentan mayores posibilidades de abastecimiento de agua. Esta condición se repite a partir del mes de julio en el cual se inicia un segundo ciclo de siembra y una nueva tendencia de incremento del índice ET/ETX. La superficie dedicada al cultivo de papa en Bailadores está alrededor de las 50 ha y el valor del índice de productividad mensual no llega a alcanzar su máximo. Esta condición limita la expansión de las siembras de papa requiriéndose la aplicación de riego suplementario. Lo anterior podría favorecer el incremento de áreas sembradas con otros cultivos de menor requerimiento hídrico.

En la Figura 4 se observa que las épocas de siembra tradicionales para el cultivo de papa en Timotes, son los meses de marzo y junio, los cuales son seguidos por períodos de 90 a 120 días con los mejores índices de ET/ETX. La superficie cultivada con papa es de aproximadamente 60 ha y a pesar de que es posible el establecimiento del cultivo durante otros meses del año, existe tradición por una gran diversidad de hortalizas con importante demanda nacional.

En la Figura 5, se presentan los índices de productividad para la localidad de Mucurubá, definiéndose dos épocas adecuadas para iniciar las plantaciones de papa como son los meses de marzo y julio, a partir de los cuales se presenta un incremento sostenido de ET/ETX durante todo el ciclo del cultivo.

En la localidad de Canaguá (Figura 6), el período de establecimiento de papa se inicia en abril y se extiende hasta el mes de agosto, con un suplemento hídrico óptimo a lo largo de casi todo el año. Para estas dos últimas localidades no se disponía de información sobre las superficies tradicionales de siembra por parte de los productores. 
Para Canaguá, el modelo genera índices de productividad mensual superiores a 0.8 durante ocho meses consecutivos (abril a noviembre). Lo anterior permitiría dos ciclos de siembra con buen suplemento de humedad, recomendándose un adecuado manejo fitosanitario por las condiciones de humedad prevalecientes. 

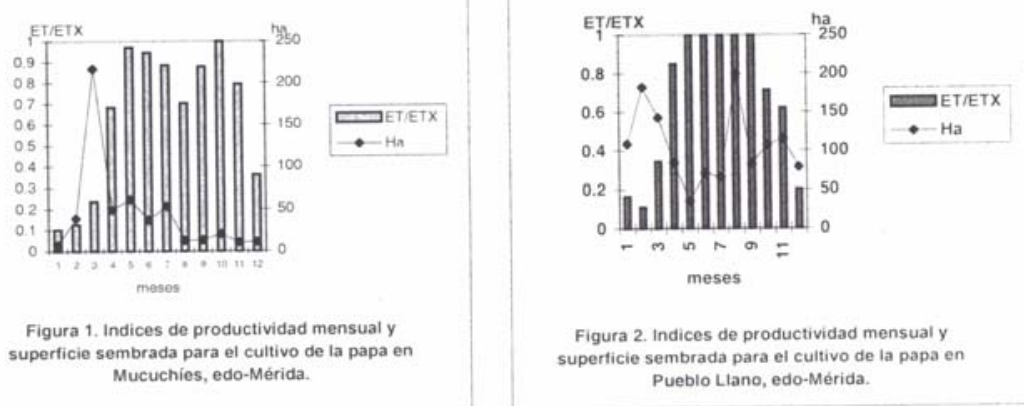

Figura 2. Indices de productividad mensual y superticie sembrada para el cultivo de la papa en Pueblo Llano, edo-Mérida.

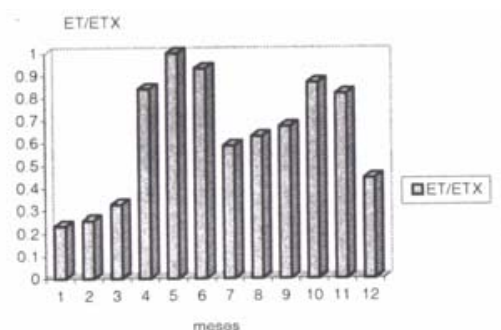

Figura 5. Indices de productividad mensual para el cultivo de la papa en Mucurubă, edo-Mérida.

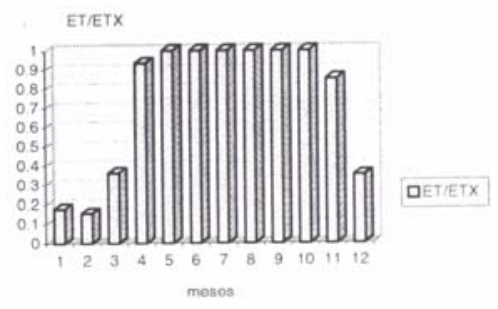

Figura 6. Indices de productividad mensual para el cultivo de la papa en Canaguá, edo-Mérida. 


\section{Conclusiones}

El modelo de balance hídrico utilizado, generó índices de productividad mensual de papa los cuales coincidieron con las épocas tradicionales de inicio del cultivo en la localidad de Pueblo Llano.

Las localidades con los mejores índices de productividad mensual consecutivos, fueron Pueblo Llano y Canaguá, condición que permitiría dos ciclos de plantación por año y garantizaría el suplemento de humedad durante las subsiguientes fases fenológicas del cultivo.

Las localidades de Bailadores y Timotes, presentaron una irregular distribución de la humedad durante todo el año, por lo tanto se requiere de la aplicación de riego suplementario para garantizar la evapotranspiración máxima en el cultivo de papa.

En las localidades de Mucuchíes y Mucurubá, el comportamiento del índice de productividad mensual presentó una tendencia con déficits de humedad durante un período de cuatro meses consecutivos con valores de ET/ETX inferiores a 0.5. Este período se considera restrictivo para iniciar las siembras si no se dispone de suministro de agua adicional.

\section{Referencias Bibliográficas}

1. Grassi, CJ. 1966. Variación de la evapotranspiración relativa durante el ciclo vegetativo de los cultivos. Memorias de las II Jornadas venezolanas de riego. Caracas, Venezuela.

2. Grassi, CJ. 1988. Fundamentos del riego. CIDIAT, Mérida.

3. Gutiérrez, O. 1975. Introducción a la evaporación y a la evapotranspiración. CIDIAT. Mérida, Venezuela.

4. Norero, A. 1975 C. Material de docencia. Postgrado de Suelos bajo riego. CIDIAT. Mérida, Venezuela.

5. Norero, A. 1982. Cálculos agronómicos relacionados con el riego. Serie Riego y Drenaje No. 28. CIDIAT, Mérida, Venezuela, p. 1725. 
6. Norero, A. 1983. Ingeniería de cultivos. CIDIAT, Mérida, Venezuela.

7. Norero, A. 1980. El agua y el aire en el suelo. Serie Suelos y Clima No. 32. CIDIAT. Mérida, Venezuela.

8. Norero, A. 1980. La fitósfera, el ambiente físico y las plantas cultivadas. Serie Suelos y Clima No. 17. CIDIAT. Mérida, Venezuela.

9. Norero, A. 1983. Crecimiento y desarrollo de cultivos anuales. Bases agrofísicas para modelos de simulación. CIDIAT, Mérida, Venezuela.

10. Sellers, N. 1965. Physical climatology. The University of Chicago Press. Chicago. USA.

11. .............. 1992. Informe Anual. FONAIAP - CIAE - Mérida, Venezuela.

12. ............... 1996. Estadísticas del sector agrícola del estado Mérida. UEDA -MAC - Mérida. Dir. de Planificación y Estadística.

13. .............. Información climatológica estado Mérida. MARNR. Dir. Hidrología. Zona 12. Mérida, Venezuela, (mimeografiado). s/f.

14. ............ 1993. Informe Anual. Estación meteorológica de Mucuchíes. FONAIAP, Mérida. Venezuela, (mimeografiado). 